This item was submitted to Loughborough's Research Repository by the author.

Items in Figshare are protected by copyright, with all rights reserved, unless otherwise indicated.

\title{
Modelling and control of a high redundancy actuator
}

PLEASE CITE THE PUBLISHED VERSION

PUBLISHER

(C) Elsevier

VERSION

AM (Accepted Manuscript)

LICENCE

CC BY-NC-ND 4.0

\section{REPOSITORY RECORD}

Du, Xinli, Roger Dixon, Roger M. Goodall, and Argyrios C. Zolotas. 2019. "Modelling and Control of a High Redundancy Actuator". figshare. https://hdl.handle.net/2134/5917. 
This item was submitted to Loughborough's Institutional Repository (https://dspace.lboro.ac.uk/) by the author and is made available under the following Creative Commons Licence conditions.

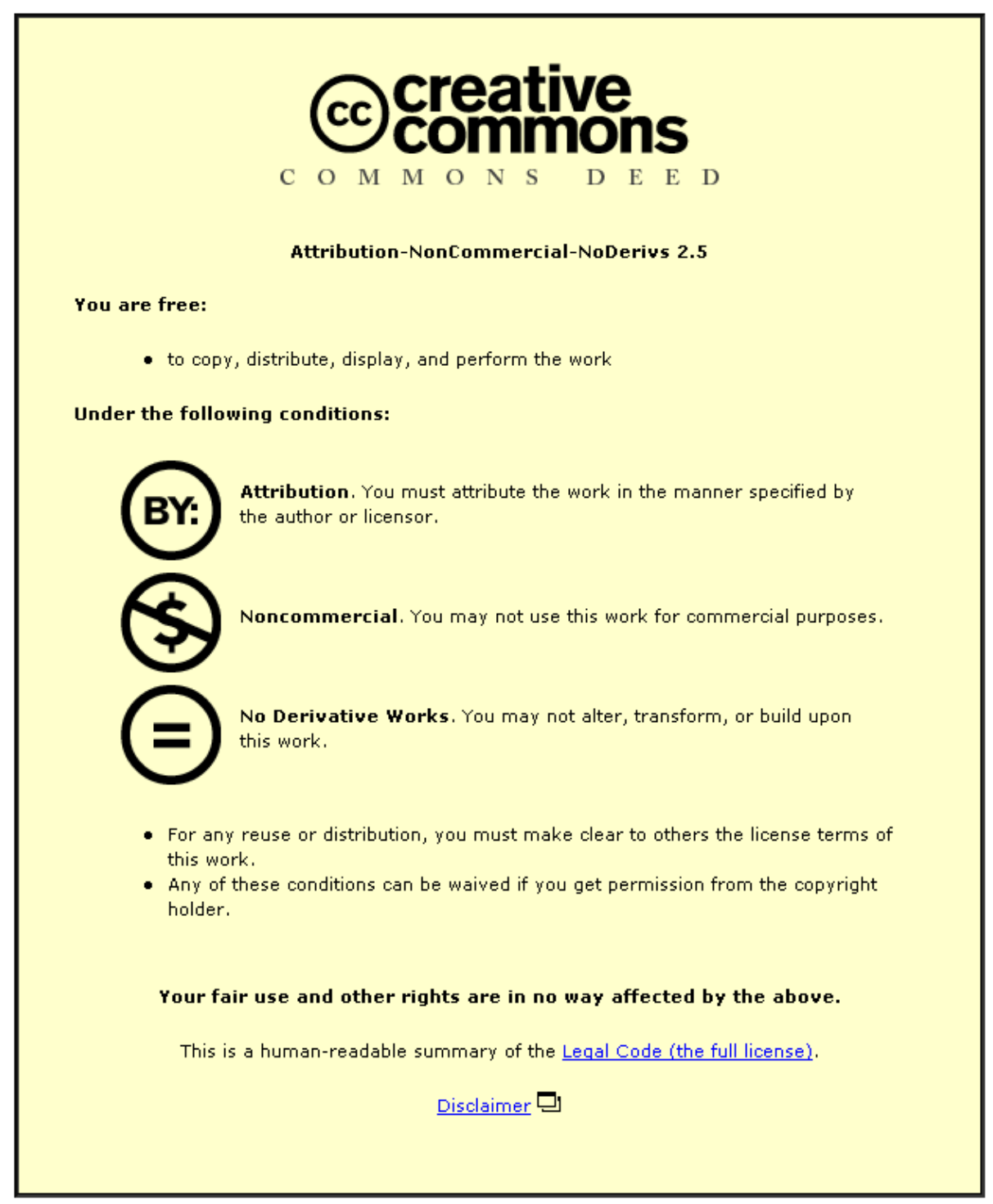

For the full text of this licence, please go to: http://creativecommons.org/licenses/by-nc-nd/2.5/ 


\title{
MODELLING AND CONTROL OF A HIGH REDUNDANCY
}

\author{
ACTUATOR \\ X. Du, R. Dixon, R. M. Goodall, and A. C. Zolotas \\ Control Systems Group, Department of Electronics and Electrical Engineering, \\ Loughborough University, Loughborough, Leicestershire, LE11 3TU, U.K. \\ Tel.+44 (0)1509 227015, Email:x.du@lboro.ac.uk
}

\begin{abstract}
The high redundancy actuation concept is a completely new approach to fault tolerance, and it is important to appreciate that it provides a transformation of the characteristics of actuators so that the actuation performance (capability) degrades slowly rather than suddenly failing, even though individual elements themselves fail. This paper aims to demonstrate the viability of the concept by showing that a highly redundant actuator, comprising a relatively large number of actuation elements, can be controlled in such a way that faults in individual elements are inherently accommodated, although some degradation in overall performance will inevitably be found. The paper introduces the notion of fault tolerant systems and the highly redundant actuator concept. Then a model for a two by two configuration with electromechanical actuation elements is derived. Two classical control approaches are then considered based on frequency domain techniques. Finally simulation results under a number of faults show the viability of the approach for fault accommodation without reconfiguration.
\end{abstract}

Keywords: fault tolerance, high redundancy, classical control

Email addresses: x.du@aston.ac.uk (Xinli Du),

r.dixon@lboro.ac.uk (Roger Dixon),

r.m.goodall@lboro.ac.uk (Roger M. Goodall),

a.c.zolotas@lboro.ac.uk (Argyrios Zolotas). 


\section{Introduction}

High levels of availability and reliability are important objectives for the design of most modern engineering systems. This is particularly true in the case of safety critical applications. Hence engineers aim to produce fault tolerant designs (this applies to both the hardware/mechanisms and the control design). The result should be fault tolerant systems, which have the capability of tolerating component malfunctions whilst still maintaining desirable and robust performance and stability properties [1]. In control terms this fault tolerance is often, but not exclusively, attained by some degree of re-configuration: i.e., detect the fault, reconfigure the system.

Practical examples of fault tolerant systems can be found in aerospace systems, e.g. Airbus fly-by-wire system [2], and Boeing 737 trailing edge flap drive system [3]. Here, low levels of functional redundancy in sensors and actuators (e.g. triplex and quadruplex) and in the control computers can be used to provide the system with the capability of fault tolerance, thereby ensuring the stability and safety of the whole system. In the above approaches, redundancy is used for fault detection and isolation (FDI) based on the continuous comparison of the system under analysis with another system characterized by the same behaviour and working in the same conditions [4]. Once a fault is detected the redundancy is then used to provide the extra control channels for the reconfiguration.

The drive-by-wire systems now have been widely used not only in aeroplane industry. The drive-by-wire systems are complex integrated systems with electronic sensors, actuators, micro-computers, information processing for single components, and engine, drive-train, suspension, and brake systems. Developed from driver-assisting systems, traditional electrically driven components, 
e.g. electronic pedal and electrically driven throttle or injection, are rendered fail-safe by the mechanics

[5]. The disadvantages of mechanical backup systems are that they are costly, heavy, passive safety-critical, and do not provide enough freedom to tap the potential of the electrical systems. Drive-by-wire systems without mechanical backup generate electrical commands through the driver and transfer them to computer-controlled electromechanical actuators, a scheme that is usually not fail-safe but has fault-tolerant properties [5].

A general fault-tolerant system comprising FDI and reconfiguration (R) scheme (as proposed in [1]) is illustrated in Fig. 1. The solid lines represent signal flow, and the dashed lines represent adaptation. The supervision system will reconfigure the actuator and/or sensor sets, and adapt the controller, based on the faults information collected by the FDI unit together with the inputs and outputs of the system, to accommodate the fault effects, generally though redundancies which are connected, usually in parallel. However a possible disadvantage of such approaches is the possibility of faults occurring in the FDI unit and the supervision system. One might ask the question, "Who/what monitors the monitors?".

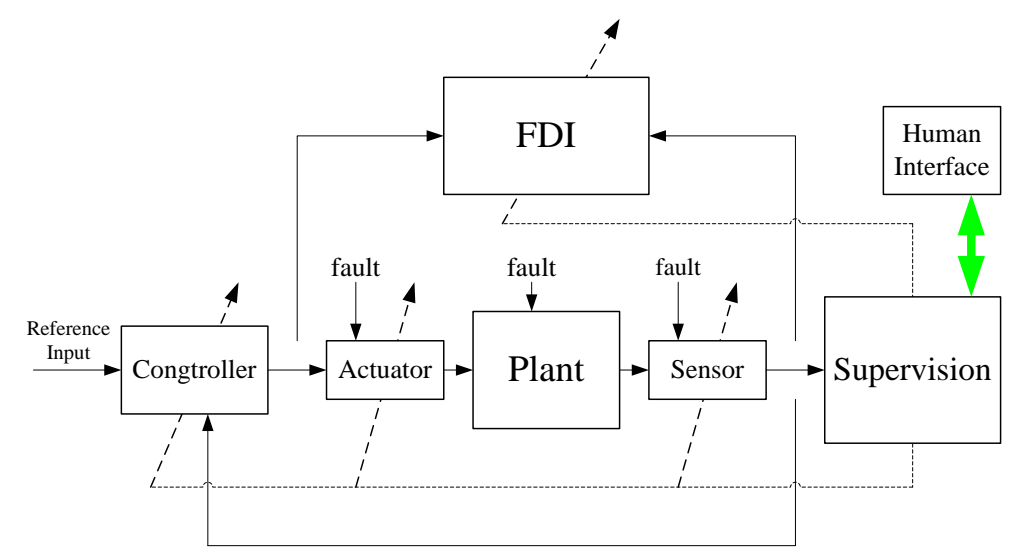

Fig. 1 Scheme of fault-tolerant control system with supervision subsystem (Patton, 1997) 
This paper proposes an alternative route to fault tolerant actuation compared with the approaches discussed above. The high redundancy actuator (HRA) suggested comprises a relatively large number of actuation elements in a matrix-like structure comprising not only parallel structure but also series structure. Different from some other fault tolerant control design against actuation failures using active method, i.e. reconfiguration $[6,7,8]$ and adaptive algorithms $[9,10]$, faults in individual actuation elements in the HRA are inherently accommodated through mechanical redundancy structure and robust control. In this design, the FDI unit may still be used for monitoring, but it is no longer strictly necessary and no reconfiguration of the controller or hardware will be needed. Instead the actuation elements work together to complete the system's objective by using the redundancy which is inherent in the structure. The HRA provides the fault tolerant ability to a component level. It combines redundancies not only in parallel, but also in series, and makes them working together under both healthy and faulty situations. When faults are injected into actuation elements, performance degradation can be expected. In practical design, it is generally unreasonable to expect a handicapped system to perform as effectively as when it is healthy. For example, the degraded performance for dynamic behaviours for an aircraft may include slower climbing rate, wider turns, slower acceleration, and other manoeuvrability [11]. For the HRA in a tracking system, the performance degradation could includes longer rise time, longer settling time, bigger overshot, but the main purpose should still be achieved, i.e. zero steady state error. If this method can be proven to work, the advantage would arise through removing the possibility of faults occurring within the FDI unit and supervision system.

Several possible structures are shown in Fig. 2. At this stage no attempt has been made to find the optimal structure for the actuators, nor have the authors attempted to identify the "best" technology for 
the actuators. Instead the research has focused on modelling and control of a relatively simple ( 2 by 2 ) actuator with a view to discovering whether controlling such structures without reconfiguration is viable. Hence, the paper is structured as follows: work on modelling is presented in section 2; the control design using a classical approach is covered in section 3; then in section 4 a selection of simulation results is presented to give an indication of performance in the presence of faults alongside the fault-free case; the paper concludes in section 5, including comments on the future direction of this research.

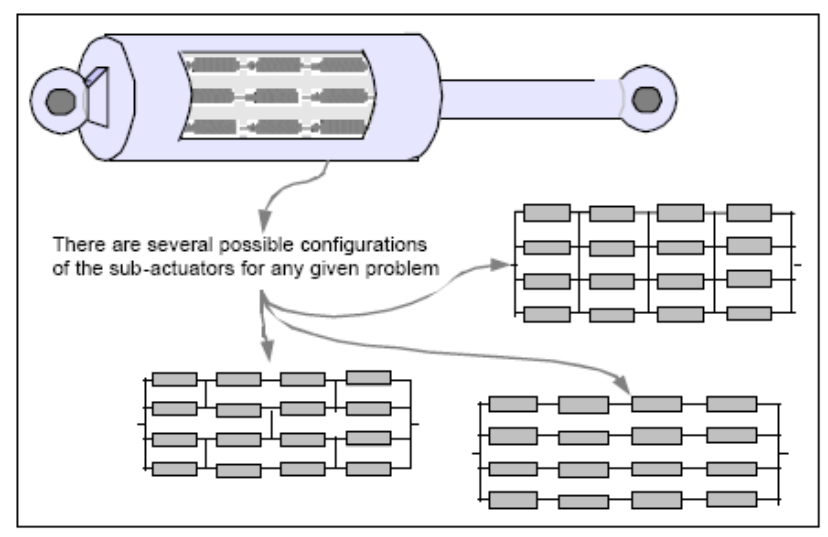

Fig. 2 Several possible configurations of the high redundancy actuator

\section{Modelling}

This section begins with modelling of an individual electro-mechanical actuator (the sub-actuation element inside the HRA). [An electro-mechanical actuation is chosen as the technology for this study because it is used in many industry applications, including manufacturing, process plant, railway vehicles and aerospace systems.] Next, four fault models which can affect electro-mechanical actuator will be introduced. Finally, in section 2.3, a model of a four elements two-by-two actuator will be constructed. 


\subsection{Modelling of individual actuator}

The electro-mechanical actuator can be considered as having two parts. One is the D.C. motor, which generates the torque, and the other is the mechanical structure (screw and nut), which translates the torque generated by the motor to the load. A schematic representation of the actuator with a load is given in Fig 3, and the free body diagram is shown in Fig. 4.

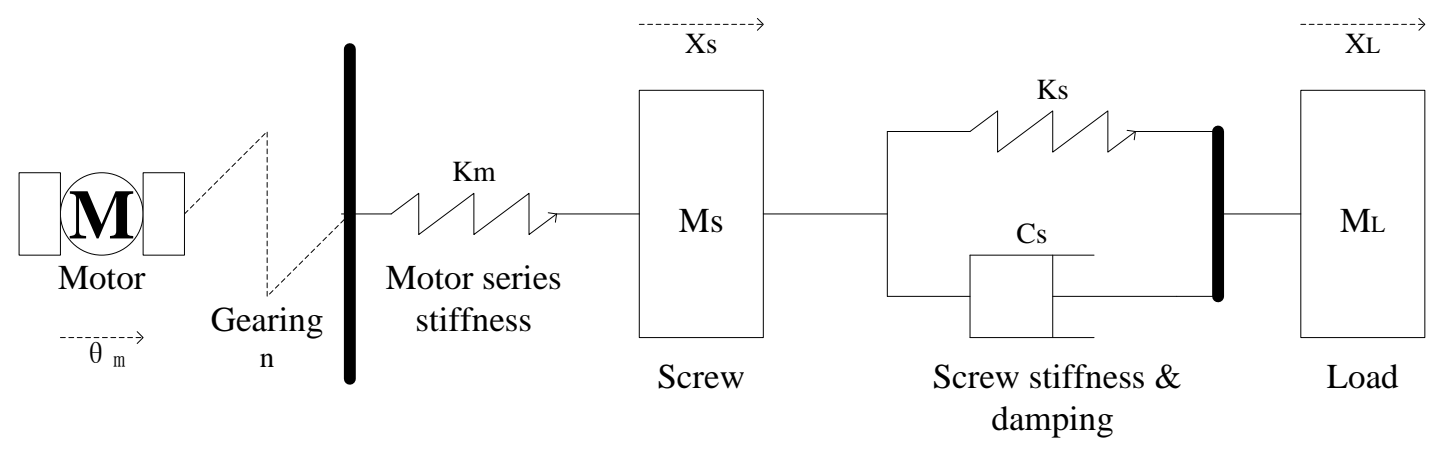

Fig. 3 Equivalent model of individual electro-mechanical actuator
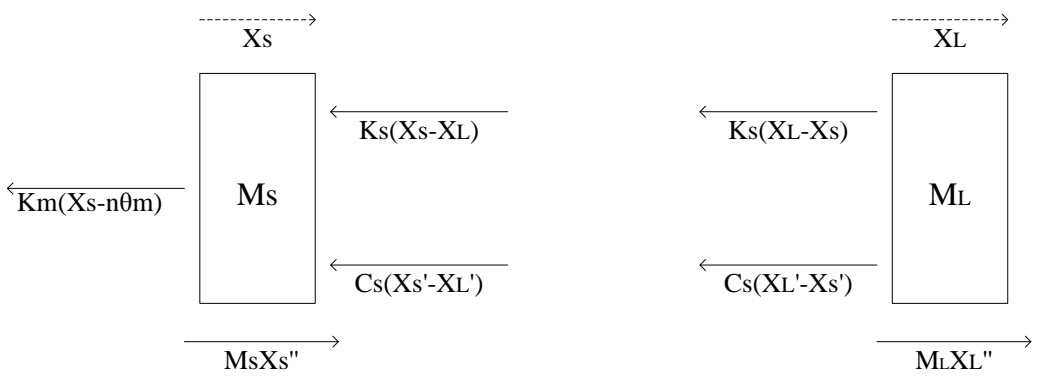

Fig. 4 Free body diagram of individual electro-mechanical actuator

On the mechanical structure, $\theta_{m}$ is the angular rotation of the motor shaft, $n$ is the screw pitch, $X_{m}$ and $X_{L}$ are positions of the screw and the end-of-actuator (load) respectively. Although the screw and nut mechanism will normally be very stiff, it does possess finite stiffness and damping so these are included in the model. $K_{m}$ represents the motor stiffness, $K_{s}$ and $C_{s}$ are the screw stiffness and 
damping respectively. $M_{s}$ and $M_{L}$ are the masses of the screw and the load.

From the free body diagram, it's straightforward to obtain the differential equations:

$$
\left\{\begin{array}{l}
M_{s} X_{s}^{\prime \prime}+K_{m}\left(X_{s}-n \theta_{m}\right)+K_{s}\left(X_{s}-X_{L}\right)+C_{s}\left(X_{s}^{\prime}-X_{L}^{\prime}\right)=0 \\
M_{L} X_{L}^{\prime \prime}+K_{s}\left(X_{L}-X_{s}\right)+C_{s}\left(X_{L}^{\prime}-X_{s}^{\prime}\right)=0
\end{array}\right.
$$

Within the D.C. servo motor, an electrical torque $t_{e}$ is generated because of the electromagnetic induction. The field flux is assumed constant so that it is proportional to the armature current $i_{a}$, while it also induces a back e.m.f., which is directly proportional to the speed $\dot{\theta}_{m}$. Hence $t_{e}=K_{t} i_{a}$ and $e_{r}=K_{e} \dot{\theta}_{m}$, where $K_{e}$ is the voltage constant depending on the number of armature turns and $K_{t}$ is the torque constant which usually takes the same value as the voltage constant. The generated electromagnetic torque will be balanced by the load torque $t_{l}$, the inertial torque $t_{a}$, and the damping torque $t_{d}$, i.e. $t_{e}=t_{l}+t_{a}+t_{d}$, where $t_{a}=J_{m} \ddot{\theta}_{m}, t_{d}=C_{m} \dot{\theta}_{m}$, and $t_{l}=K_{m}\left(\theta_{m} n-x_{m}\right) n$ which is introduced from the load. $J_{m}$ accounts for motor inertia and $C_{m}$ accounts for the damping coefficient. The applied voltage relationship is given in the following equations, where $R_{a r m}$ is the resistance and $L_{a r m}$ is the inductance of the windings:

$$
\left\{\begin{array}{l}
K_{t} i_{a}-K_{m}\left(\theta_{m} n-X_{m}\right) n-J_{m} \dot{\omega}_{r}-C_{m} \omega_{r}=0 \\
v_{a}-R_{a r m} i_{a}-L_{a r m} i_{a}-K_{e} \dot{\theta}_{m}=0
\end{array}\right.
$$

Combining the electrical (2.2) with the mechanical (2.1) equations, a linear model can be developed in MATLAB/Simulink as presented in Fig 5. 


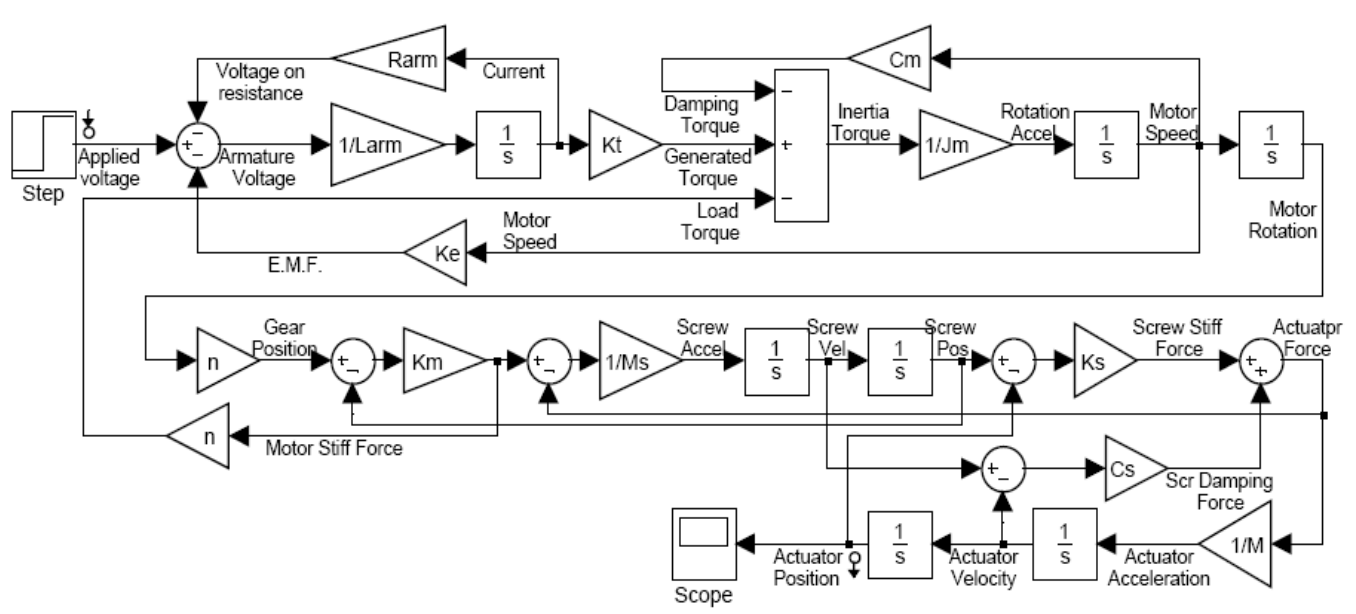

Fig. 5 Linear model of individual electro-mechanical actuator

At the output of the motor model, motor rotation is given. It is transformed by a gearing factor which provides a linear motion to the screw. The output of the actuator is a force and this force is generated by compression of the screw as the motor rotates. The actuator (and load) velocity and position are obtained based on Newton's second law, and they are fed back to the actuator model to calculate the output force.

The Simulink model is used in the time domain simulation and allows fault injection. However, to facilitate control design (in MATLAB), it is also possible to obtain the linear model in the state-space form: $\dot{x}=A x+B u, y=C x$.

The state vector is chosen as $x=\left[\begin{array}{lllllll}\dot{i}_{a} & \dot{\theta}_{m} & \theta_{m} & \dot{X}_{m} & X_{m} & \dot{X}_{L} & X_{L}\end{array}\right]^{T}$, and the input $u$ and output $y$ are chosen as $u=v_{a}, y=X_{L}$, where $v_{a}$ is the applied voltage. The state transition, input and observation matrices for the model are: 


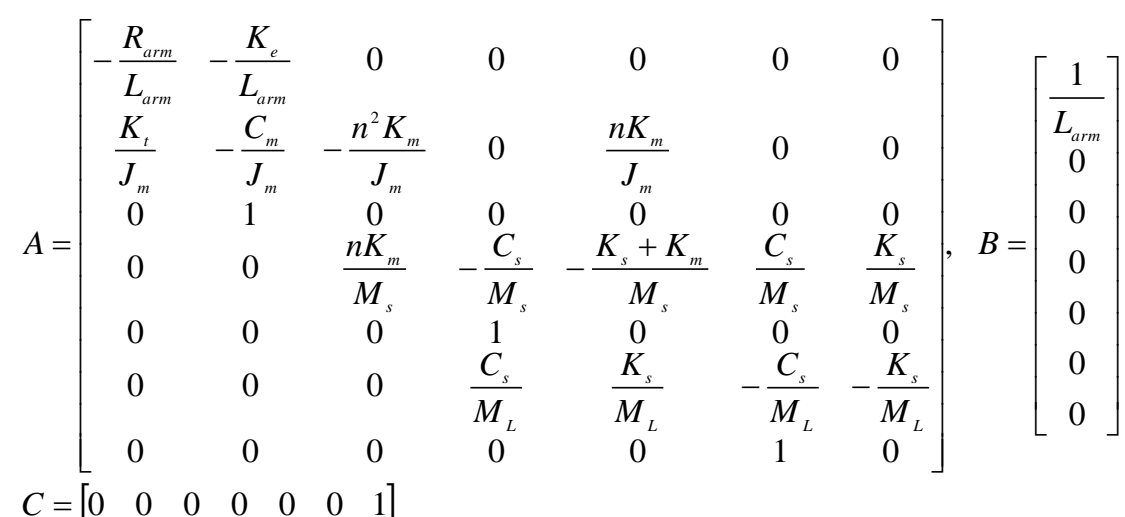

The meaning and value of the parameters and states are listed in Table 1. The motor parameters come from a real D.C. servo motor provided by McLennan Servo Supplies Ltd, Industrial DC Servo Motor M543E [12]. The other mechanical parameters are derived from [13].

TABLE 1

DEFINITION OF PARAMETERS AND STATES IN STATE SPACE MODEL

\begin{tabular}{|c|c|c|c|c|c|}
\hline$R_{\text {arm }}$ & motor resistance & $2.2 \Omega$ & $L_{\text {arm }}$ & inductance of the windings & $6.4 \mathrm{mH}$ \\
\hline$K_{e}$ & voltage constant & $0.121 \mathrm{~V} / \mathrm{rad} / \mathrm{s}$ & $K_{t}$ & torque constant & $0.121 \mathrm{Nm} / \mathrm{A}$ \\
\hline$J_{m}$ & motor inertia & $5.3 \mathrm{e}-5 \mathrm{kgm}^{\wedge} 2$ & $C_{m}$ & damping coefficient & $8 \mathrm{e}-5 \mathrm{Nm} / \mathrm{rad} / \mathrm{s}$ \\
\hline$K_{m}$ & motor stiffness & $1 \mathrm{e} 7 \mathrm{~N} / \mathrm{m}$ & $n$ & screw pitch & $3.82 \mathrm{e}-4 \mathrm{~m} / \mathrm{rad}$ \\
\hline$K_{s}$ & screw stiffness & $1.8 \mathrm{e} 5 \mathrm{~N} / \mathrm{m}$ & $C_{s}$ & Screw damping & $1.2 \mathrm{e} 3 \mathrm{~N} / \mathrm{m} / \mathrm{s}$ \\
\hline$M_{s}$ & screw mass & $2 \mathrm{~kg}$ & $M_{L}$ & load mass & $20 \mathrm{~kg}$ \\
\hline$i_{a}$ & armature current & A & $\theta_{m}$ & angular rotation of the motor shaft & $\mathrm{rad}$ \\
\hline$X_{m}$ & screw position & $\mathrm{m}$ & $X_{L}$ & end-of-actuator position & $\mathrm{m}$ \\
\hline
\end{tabular}

The actuator faults can be divided into two types: motor faults which occur in the D.C. motor as electrical faults, and the mechanical faults which happen in the components where the torque is 
transferred to the load. The faults considered are summarised below.

\subsection{Individual actuator faults}

Overheating: This may either be caused by insulation breakdown leading to shorted turns, or to some field demagnetisation. In both cases it will cause reduction in the voltage constant $K_{e}$ and the torque constant $K_{t}$.

Open circuit: The effect is that the resistance of the rotor will be very large so that almost no current will flow. Hence no torque will be generated by the motor.

Backlash: Generally, the backlash effect occurs due to wear in the screw/nut mechanism. Its effect can be described using a dead-zone [14].

Lockup: Excessive wear in the screw/nut mechanism may create mechanical interference within the mechanism such that the actuator becomes locked. This fault is serious as it can not be solved (by reconfiguration) at the signal level.

\subsection{Modelling of two by two configuration}

Having developed the basic (sub-actuator) model, it is relatively straightforward to build multi-actuator systems by combining the individual sub-actuators. There are two basic configurations to connect two individual actuators, one is in series and the other is in parallel. In the series configuration, one actuator is connected at the end of the other thereby doubling the extension length and velocity. In the parallel configuration, two actuators are connected to push/pull the same load so that the force output is doubled. Taking advantage of both these connecting structures, two separate actuators can be 
developed and modelled. The first one is "parallel-in-series" (PS), as shown in the left of Fig. 6, and the other one is denoted "series-in-parallel" (SP), as shown in the right of Fig. 6.

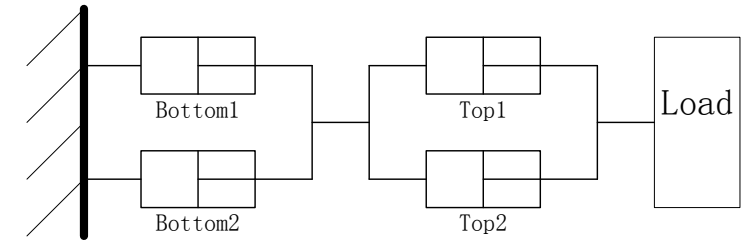

(a) parallel-in-series

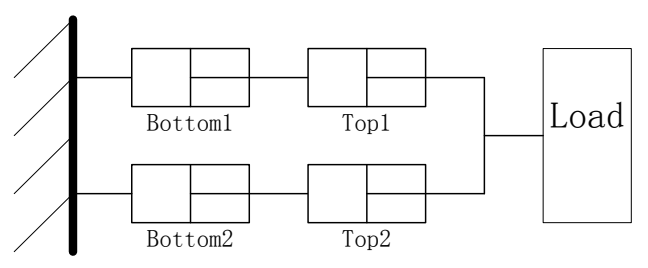

(b) series-in-parallel

Fig. 6 Two kinds of two by two configurations

In a parallel structure, the elements are connected together at the end so that the structure will fail fixed when one of them is locked up. The PS configuration comprises two parallel structures while the SP only has one, so it will reduce the reliability under a lockup fault situation. For the present study the SP configuration is adopted because of its greater reliability. The development of a model of the SP configuration begins with the equivalent model of Fig 7 and the free body diagram shown in Fig 8 .

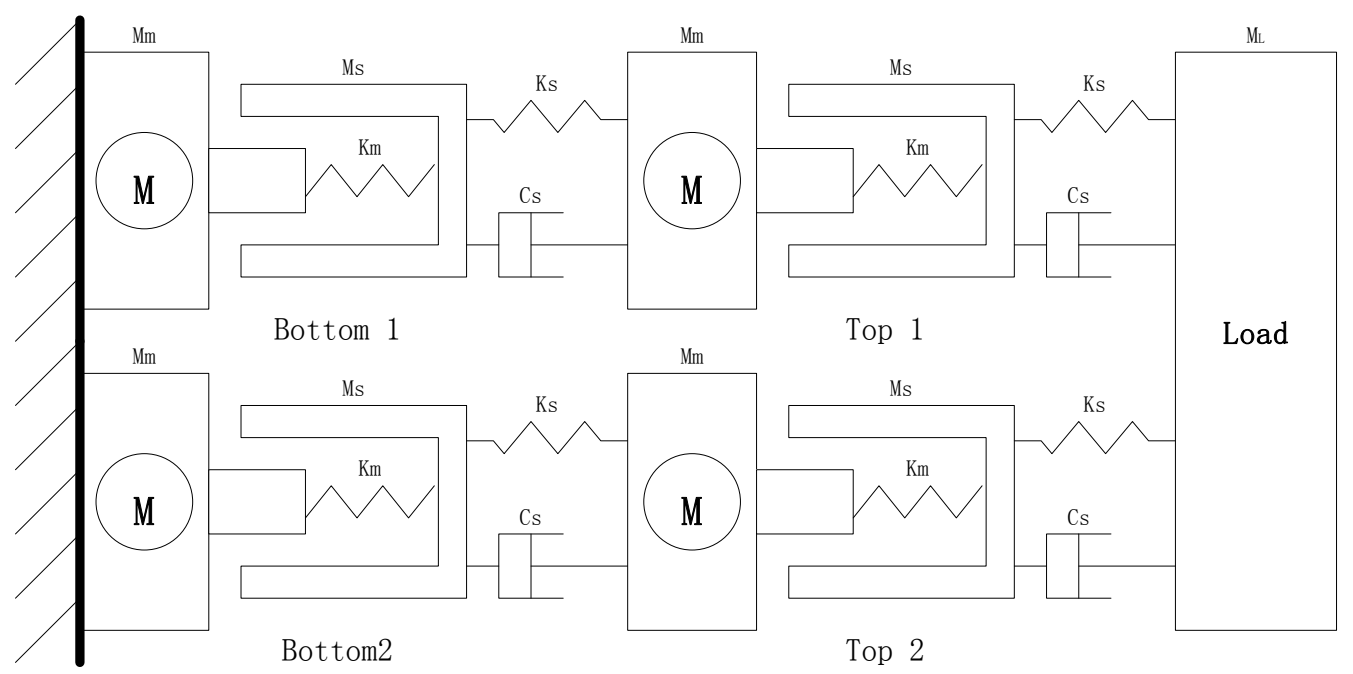

Fig. 7 Equivalent model of two by two SP configuration 


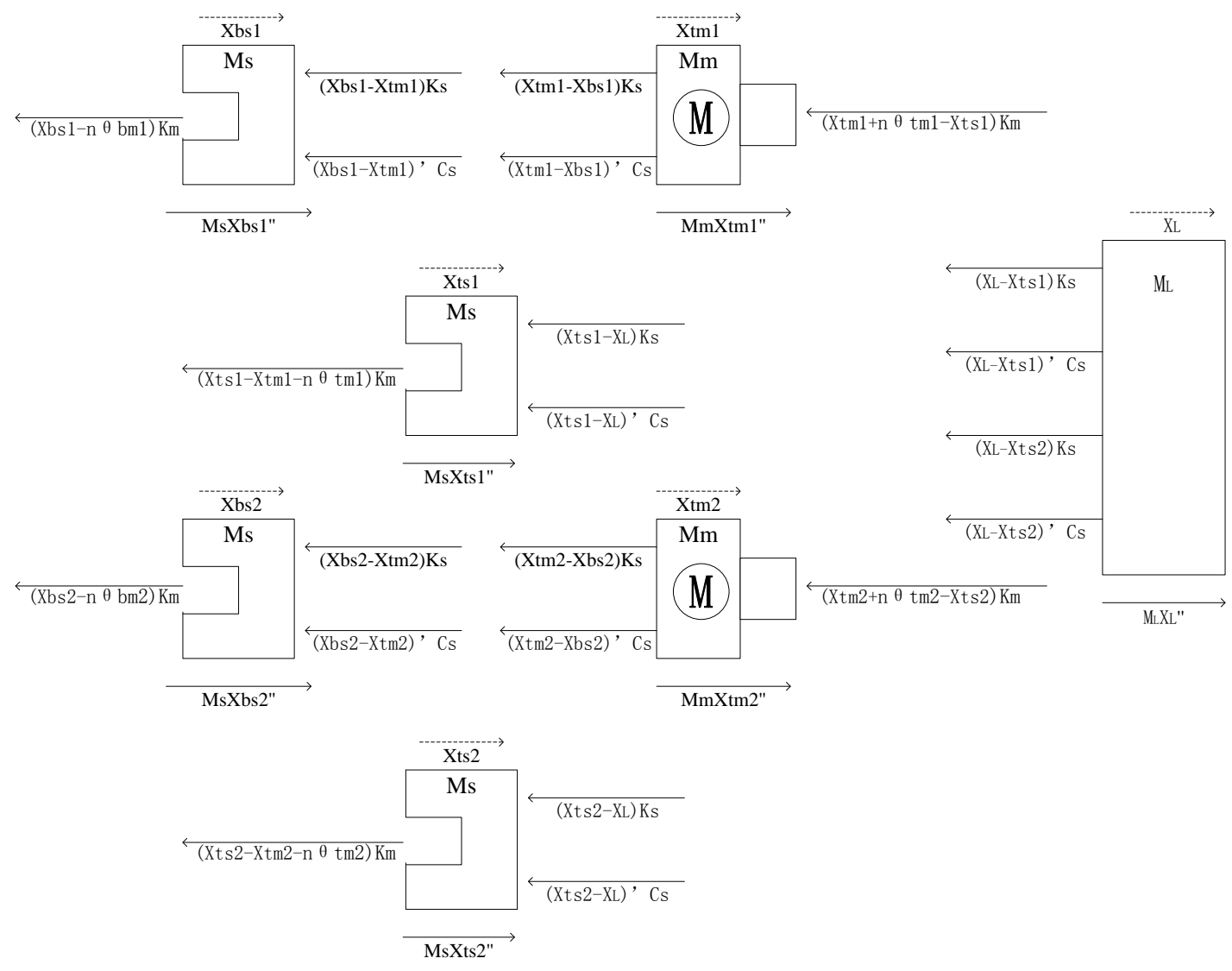

Fig. 8 Free body diagram of two by two SP configuration

In the SP configuration, the top actuator connected to the bottom actuator is seen as part of the load. In

Fig $8, M_{m}$ represents the mass of the motor; the subscript, b1 and b2 denote bottom actuator 1 and 2 respectively; $\mathrm{t} 1$ and $\mathrm{t} 2$ denote top actuator 1 and 2 as described in the equivalent model. According to the free body diagram, the following representation of the mechanical part can be obtained:

$$
\left\{\begin{array}{l}
M_{s} X_{b s 1}^{\prime \prime}+\left(X_{b s 1}-n \theta_{b m 1}\right) K_{m}+\left(X_{b s 1}-X_{t m 1}\right) K_{s}+\left(X_{b s 1}^{\prime}-X_{t m 1}^{\prime}\right) C_{s}=0 \\
M_{m} X_{t m 1}^{\prime \prime}+\left(X_{t m 1}-X_{b s 1}\right) K_{s}+\left(X_{t m 1}^{\prime}-X_{b s 1}^{\prime}\right) C_{s}+\left(X_{t m 1}+n \theta_{t m 1}-X_{t s 1}\right) K_{m}=0 \\
M_{s} X_{t s 1}^{\prime \prime}+\left(X_{t s 1}-X_{t m 1}-n \theta_{t m 1}\right) K_{m}+\left(X_{t s 1}-X_{L}\right) K_{s}+\left(X_{t s 1}^{\prime}-X_{L}^{\prime}\right) C_{s}=0 \\
M_{s} X_{b s 2}^{\prime \prime}+\left(X_{b s 2}-n \theta_{b m 2}\right) K_{m}+\left(X_{b s 2}-X_{t m 2}\right) K_{s}+\left(X_{b s 2}^{\prime}-X_{t m 2}^{\prime}\right) C_{s}=0 \\
M_{m} X_{t m 2}^{\prime \prime}+\left(X_{t m 2}-X_{b s 2}\right) K_{s}+\left(X_{t m 2}^{\prime}-X_{b s 2}^{\prime}\right) C_{s}+\left(X_{t m 2}+n \theta_{t m 2}-X_{t s 2}\right) K_{m}=0 \\
M_{s} X_{t s 2}^{\prime \prime}+\left(X_{t s 2}-X_{t m 2}-n \theta_{t m 2}\right) K_{m}+\left(X_{t s 2}-X_{L}\right) K_{s}+\left(X_{t s 2}^{\prime}-X_{L}^{\prime}\right) C_{s}=0 \\
M_{l} X_{L}^{\prime \prime}+\left(X_{L}-X_{t s 1}\right) K_{s}+\left(X_{L}^{\prime}-X_{t s 1}^{\prime}\right) C_{s}+\left(X_{L}-X_{t s 2}\right) K_{s}+\left(X_{L}^{\prime}-X_{t s 2}^{\prime}\right) C_{s}=0
\end{array}\right.
$$


The equations for each of the four D.C. motors are identical to those for the individual actuator and it is assumed that all sub-actuation elements are driven from the same power supply. Assuming that all four actuators are identical, each actuator has the same parameter values as shown in Table 1. Combining equations 2.2 and 2.4 gives a twenty-six state state-space model with the input and output being the applied voltage and position of the load respectively.

In the structure of the SP configuration, state matrix A will comprise both the model of each individual sub-actuator and the coupling between the sub-actuators, thus it can be partitioned into a $4 \times 4$ matrix based on the physical meaning with elements b1, b2 (the bottom set of sub-actuators), t1, t2 (the top set of sub-actuators) in the diagonal, and their coupling relations in the off-diagonal positions.

$A=\left[\begin{array}{cccc}b 1 & b 1 * t 1 & b 1 * b 2 & b 1 * t 2 \\ t 1 * b 1 & t 1 & t 1 * b 2 & t 1 * t 2 \\ b 2 * b 1 & b 2 * t 1 & b 2 & b 2 * t 2 \\ t 2 * b 1 & t 2 * t 1 & t 2 * b 2 & t 2\end{array}\right]_{4 * 4}$ or, $A=\left[\begin{array}{cccc}A_{11} & A_{12} & A_{13} & A_{14} \\ A_{21} & A_{22} & A_{23} & A_{24} \\ A_{31} & A_{32} & A_{33} & A_{34} \\ A_{41} & A_{42} & A_{43} & A_{44}\end{array}\right]_{4 * 4}$

In fact, the $A_{i j}$ for all $i=1,2,3,4$ and $j=1,2,3,4$ sub-matrices are given as follows:

$A_{11}=\left[\begin{array}{ccccccc}-\frac{R_{a m m}}{L_{a m m}} & -\frac{K_{e}}{L_{a m m}} & 0 & 0 & 0 & 0 & 0 \\ \frac{K_{t}}{J_{m}} & -\frac{C_{m}}{J_{m}} & -\frac{n^{2} K_{m}}{J_{m}} & 0 & \frac{n K_{m}}{J_{m}} & 0 & 0 \\ 0 & 1 & 0 & 0 & 0 & 0 & 0 \\ 0 & 0 & \frac{n K_{m}}{M_{s}} & -\frac{C_{s}}{M_{s}} & -\frac{K_{s}+K_{m}}{M_{s}} & \frac{C_{s}}{M_{s}} & \frac{K_{s}}{M_{s}} \\ 0 & 0 & 0 & 1 & 0 & 0 \\ 0 & 0 & 0 & \frac{C_{s}}{M_{L}} & \frac{K_{s}}{M_{L}} & -\frac{C_{s}}{M_{L}} & -\frac{K_{m}+K_{s}}{M_{L}} \\ 0 & 0 & 0 & 0 & 0 & 1 & 0\end{array}\right]_{7 * 7} A_{12}=\left[\begin{array}{ccccc}0 & 0 & 0 & 0 & 0 \\ 0 & 0 & 0 & 0 & 0 \\ 0 & 0 & 0 & 0 & 0 \\ 0 & 0 & 0 & 0 & 0 \\ 0 & 0 & 0 & 0 & 0 \\ 0 & 0 & -\frac{n K_{m}}{M_{m}} & 0 & \frac{K_{m}}{M_{m}} \\ 0 & 0 & 0 & 0 & 0\end{array}\right]_{7 * 5}$ $A_{13}=0_{7 * 7}, A_{14}=0_{7^{* 7} 7}$, 
$A_{21}=\left[\begin{array}{ccccccc}0 & 0 & 0 & 0 & 0 & 0 & 0 \\ 0 & 0 & 0 & 0 & 0 & 0 & -\frac{n K_{m}}{J_{m}} \\ 0 & 0 & 0 & 0 & 0 & 0 & 0 \\ 0 & 0 & 0 & 0 & 0 & 0 & \frac{K_{m}}{M_{s}} \\ 0 & 0 & 0 & 0 & 0 & 0 & 0\end{array}\right]_{5^{*} 7} \quad, A_{22}=\left[\begin{array}{ccccc}-\frac{R_{a r m}}{L_{a r m}} & -\frac{K_{e}}{L_{a r m}} & 0 & 0 & 0 \\ \frac{K_{t}}{J_{m}} & -\frac{C_{m}}{J_{m}} & -\frac{n^{2} K_{m}}{J_{m}} & 0 & \frac{n K_{m}}{J_{m}} \\ 0 & 1 & 0 & 0 & 0 \\ 0 & 0 & \frac{n K_{m}}{M_{s}} & -\frac{C_{s}}{M_{s}} & -\frac{K_{m}+K_{s}}{M_{s}} \\ 0 & 0 & 0 & 1 & 0\end{array}\right]_{5^{* 5}}$

$A_{23}=0_{5^{* 7}}, A_{24}=\left[\begin{array}{ccccccc}0 & 0 & 0 & 0 & 0 & 0 & 0 \\ 0 & 0 & 0 & 0 & 0 & 0 & 0 \\ 0 & 0 & 0 & 0 & 0 & 0 & 0 \\ 0 & 0 & 0 & 0 & 0 & \frac{C_{s}}{M_{s}} & \frac{K_{s}}{M_{s}} \\ 0 & 0 & 0 & 0 & 0 & 0 & 0\end{array}\right]_{5^{* 7}}$

$A_{31}=0_{7 * 7}, A_{32}=0_{7 * 5}$,

$A_{33}=\left[\begin{array}{ccccccc}-\frac{R_{a r m}}{L_{a r m}} & -\frac{K_{e}}{L_{a r m}} & 0 & 0 & 0 & 0 & 0 \\ \frac{K_{t}}{J_{m}} & -\frac{C_{m}}{J_{m}} & -\frac{n^{2} K_{m}}{J_{m}} & 0 & \frac{n K_{m}}{J_{m}} & 0 & 0 \\ 0 & 1 & 0 & 0 & 0 & 0 & 0 \\ 0 & 0 & \frac{n K_{m}}{M_{s}} & -\frac{C_{s}}{M_{s}} & -\frac{K_{s}+K_{m}}{M_{s}} & \frac{C_{s}}{M_{s}} & \frac{K_{s}}{M_{s}} \\ 0 & 0 & 0 & 1 & 0 & 0 & 0 \\ 0 & 0 & 0 & \frac{C_{s}}{M_{L}} & \frac{K_{s}}{M_{L}} & -\frac{C_{s}}{M_{L}} & -\frac{K_{m}+K_{s}}{M_{L}} \\ 0 & 0 & 0 & 0 & 0 & 1 & 0\end{array}\right]_{7 * 7} A_{34}=\left[\begin{array}{ccccccc}0 & 0 & 0 & 0 & 0 & 0 & 0 \\ 0 & 0 & 0 & 0 & 0 & 0 & 0 \\ 0 & 0 & 0 & 0 & 0 & 0 & 0 \\ 0 & 0 & 0 & 0 & 0 & 0 & 0 \\ 0 & 0 & 0 & 0 & 0 & 0 & 0 \\ 0 & 0 & -\frac{n K_{m}}{M_{m}} & 0 & -\frac{K_{m}}{M_{m}} & 0 & 0 \\ 0 & 0 & 0 & 0 & 0 & 0 & 0\end{array}\right]_{7 * 7}$

$A_{41}=0_{7^{* 7}}, A_{42}=\left[\begin{array}{ccccc}0 & 0 & 0 & 0 & 0 \\ 0 & 0 & 0 & 0 & 0 \\ 0 & 0 & 0 & 0 & 0 \\ 0 & 0 & 0 & 0 & 0 \\ 0 & 0 & 0 & 0 & 0 \\ 0 & 0 & 0 & \frac{C_{s}}{M_{L}} & \frac{K_{s}}{M_{L}} \\ 0 & 0 & 0 & 0 & 0\end{array}\right]_{7^{* 5}}$

$A_{43}=\left[\begin{array}{ccccccc}0 & 0 & 0 & 0 & 0 & 0 & 0 \\ 0 & 0 & 0 & 0 & 0 & 0 & -\frac{n K_{m}}{J_{m}} \\ 0 & 0 & 0 & 0 & 0 & 0 & 0 \\ 0 & 0 & 0 & 0 & 0 & 0 & \frac{K_{m}}{M_{s}} \\ 0 & 0 & 0 & 0 & 0 & 0 & 0 \\ 0 & 0 & 0 & 0 & 0 & 0 & 0 \\ 0 & 0 & 0 & 0 & 0 & 0 & 0\end{array}\right]_{7 * 7}, A_{44}=\left[\begin{array}{cccccccc}-\frac{R_{a r m}}{L_{a r m}} & -\frac{K_{e}}{L_{a r m}} & 0 & 0 & 0 & 0 & 0 \\ \frac{K_{t}}{J_{m}} & -\frac{C_{m}}{J_{m}} & -\frac{n^{2} K_{m}}{J_{m}} & 0 & \frac{n K_{m}}{J_{m}} & 0 & 0 \\ 0 & 1 & 0 & 0 & 0 & 0 & 0 \\ 0 & 0 & \frac{n K_{m}}{M_{s}} & -\frac{C_{s}}{M_{s}} & -\frac{K_{s}+K_{m}}{M_{s}} & \frac{C_{s}}{M_{s}} & \frac{K_{s}}{M_{s}} \\ 0 & 0 & 0 & 1 & 0 & 0 & 0 \\ 0 & 0 & 0 & \frac{C_{s}}{M_{L}} & \frac{K_{s}}{M_{L}} & -\frac{2 C_{s}}{M_{L}} & -\frac{2 K_{s}}{M_{L}} \\ 0 & 0 & 0 & 0 & 0 & 1 & 0\end{array}\right]_{7 * 7}$

and the corresponding B,C matrices are:

$$
\begin{aligned}
B & =\left[\begin{array}{cccccccccccccccccccccccccc}
\frac{1}{L_{a r m}} & 0 & 0 & 0 & 0 & 0 & 0 & \frac{1}{L_{a r m}} & 0 & 0 & 0 & 0 & \frac{1}{L_{a r m}} & 0 & 0 & 0 & 0 & 0 & 0 & \frac{1}{L_{a r m}} & 0 & 0 & 0 & 0 & 0 & 0
\end{array}\right]^{T}, \\
C & =\left[\begin{array}{llllllllllllllllllllllllll}
0 & 0 & 0 & 0 & 0 & 0 & 0 & 0 & 0 & 0 & 0 & 0 & 0 & 0 & 0 & 0 & 0 & 0 & 0 & 0 & 0 & 0 & 0 & 0 & 0 & 1
\end{array}\right]
\end{aligned}
$$




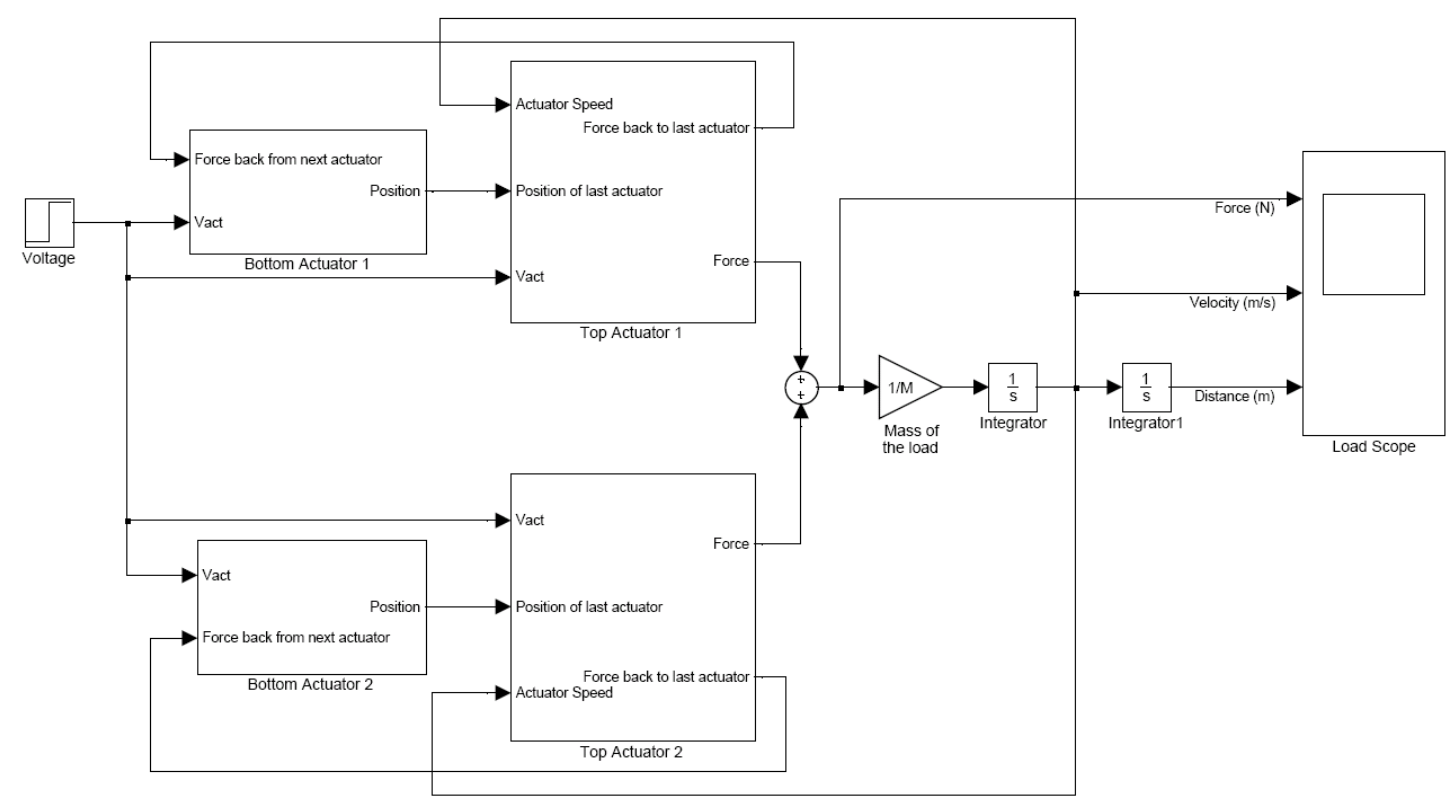

Fig. 9 Linear model of two by two SP configuration

A linear model built in MATLAB/Simulink is shown in Fig. 9. The coupling between the different actuators is clearly evident, by noting the interconnections of position and force information between the different actuators.

The aforementioned models can be used for control design and test of the closed-loop system under fault scenarios in Simulink.

\section{Control Design}

PID controllers as well as lead/lag compensator networks have been very successful classical control structures utilised in a number of industrial applications. It is rather straightforward to derive the required transfer function relationship needed for classical control design from the state space model. In particular, a position control design is followed, using two control schemes, i.e. a global controller 
for the full actuator assembly and a global controller for the full actuator assembly with local controllers at each sub-actuator, see Fig. 10.

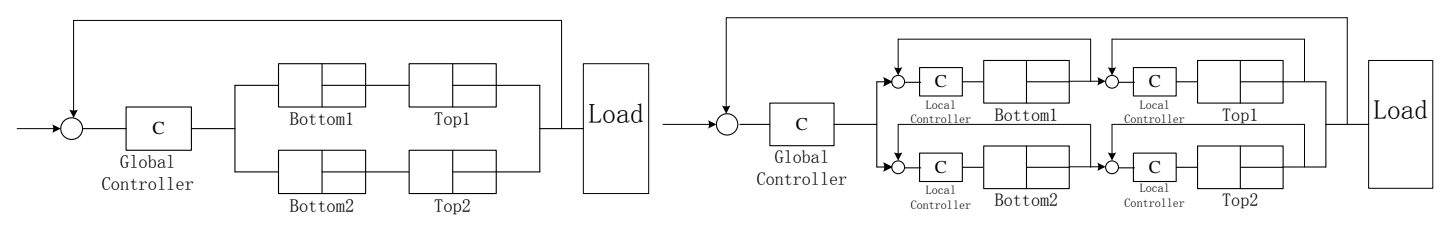

(a) voltage-driven

(b) current-driven

Fig. 10 Control structures

\subsection{Control structure}

Referring to Fig. 10 above, voltage-driven control comprises one controller based on the position output of the HRA, while current-driven control is an extension including local controllers to regulate the motor currents of each individual sub-actuator within the HRA.

Both control schemes use position feedback information for the global controller while Fig. 10(b) uses additional current feedback information for the local controllers. The two control structures are designed to provide similar performance thus enabling a fair comparison of the sensitivity to faults for both structures (see section 4).

\subsection{Controller design}

First, the voltage-driven controller is considered. Since the open-loop system gain is very small at low frequency (see Fig. 11), a proportional controller with a gain of $1000 \mathrm{~V} / \mathrm{m}$ is chosen for the global 
position controller to raise the magnitude without overloading the D.C. motor. After the compensation, the phase margin (P.M.) is $83.7 \mathrm{deg}$ and the gain margin (G.M.) is $11.4 \mathrm{~dB}$ so that no other compensator is needed. The closed-loop bandwidth is about $1 \mathrm{~Hz}$ for the overall feedback system.

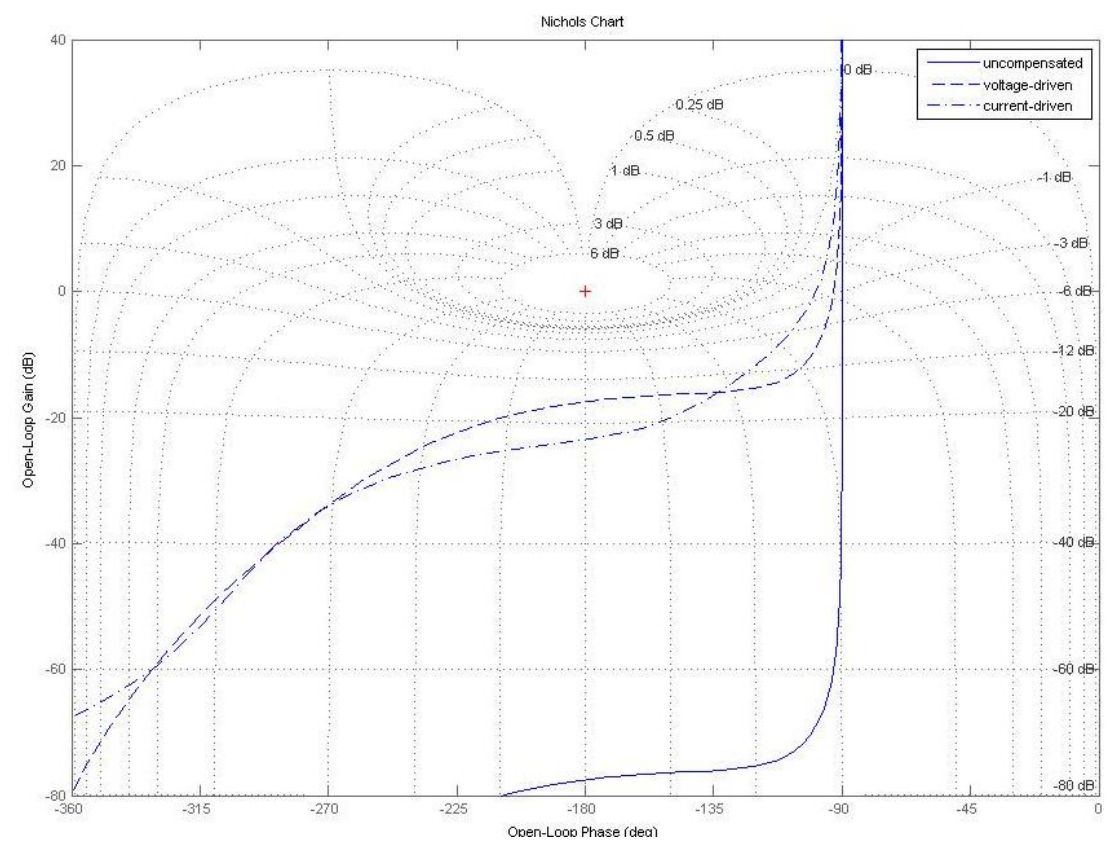

Fig. 11 Performance in frequency domain using classical control

For the structure with local current controller, generally a faster inner current loop is needed. Using integral controller with a gain of $800 \mathrm{~V} / \mathrm{A}$, the bandwidth of the current inner loop is set to about $75 \mathrm{~Hz}$. Then a lead compensator is designed for the global position controller with a gain of $156.66 \mathrm{~A} / \mathrm{m}$, a zero of -1.09 and a pole of -36.31 . With this design, the P.M. is $80.8 \mathrm{deg}$, the G.M. is $23.5 \mathrm{~dB}$, and the closed-loop bandwidth is again about $1 \mathrm{~Hz}$. A time domain response to a $0.06 \mathrm{~m}$ step command is given in Fig. 12 for both control structure without and with local current control as well as the performance in frequency domain shown in an open loop Nichols chart for the uncompensated and compensated systems in Fig. 11. 

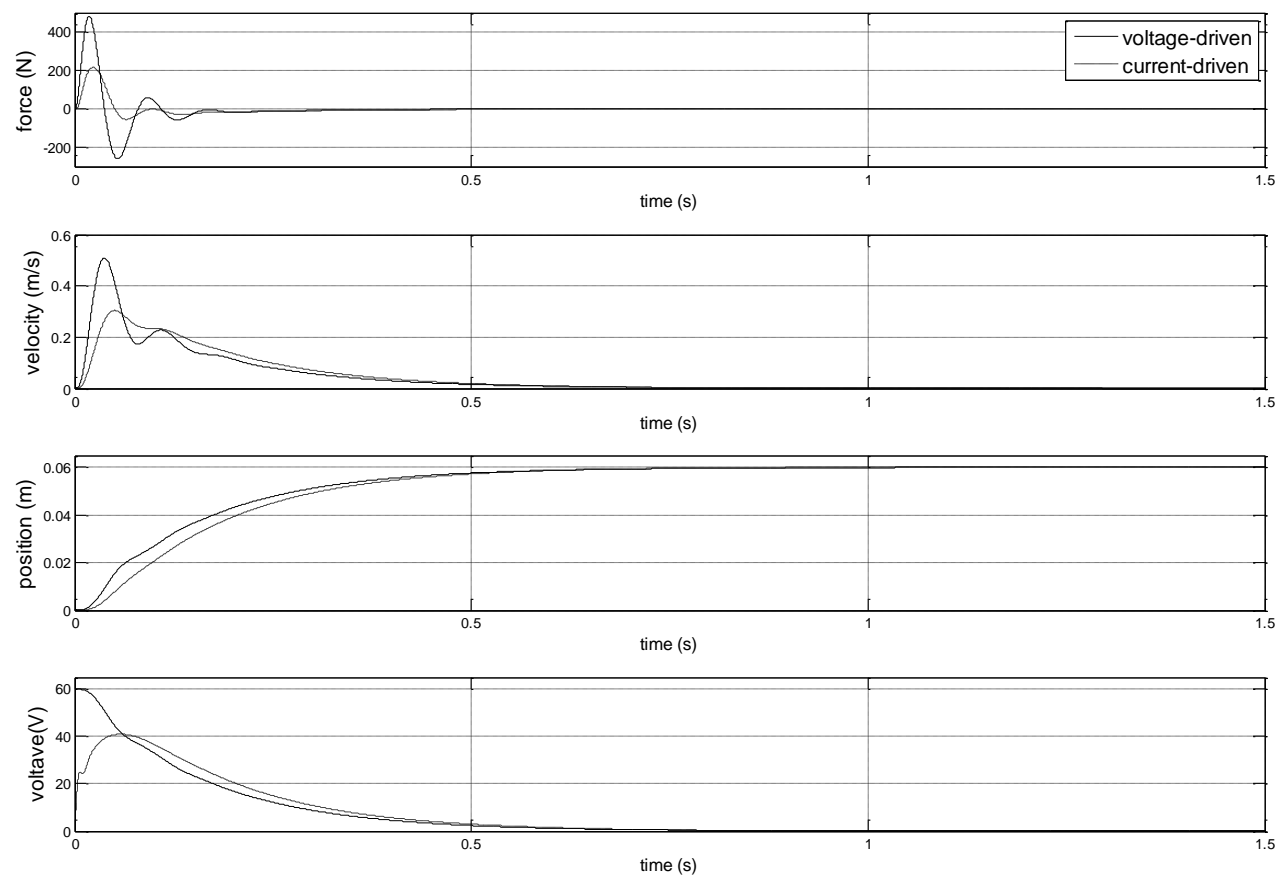

Fig. 12 Performance in time domain using classical control

Very similar performance can be seen for both voltage-driven and current-driven control structures because of the design process. But to get such similar performance, the output voltage of the current-driven controller is limited at $40 \mathrm{~V}$ while the maxim output of voltage-driven controller is $60 \mathrm{~V}$, which means the system using current-driven structure can be faster than the one has been designed. The benefit of these systems having similar performance is that the behaviour of both schemes can be compared when faults are introduced.

\section{Simulation results}

In sections 2 and 3, a two by two series-in-parallel configuration for a HRA has been modelled and controllers have been designed. In this section, the HRA is tested under fault-injected situations. Both the controller and the control structure remain unchanged when the fault is injected (i.e. there is no 
reconfiguration).

Four kinds of actuator faults are modelled: Overheating, Open circuit, Backlash and Lockup. It should be noted that lock-up (fail-fixed) and open-circuit (fail-loose) are considered to be worst cases.

The Bottom 1 sub-actuator, as shown in Fig. 6, is chosen as the fault injected element. Two kinds of control set-point input will be used for testing purpose, a step signal and a ramp signal. Both time and frequency domain performances will be compared to see how much degradation occurs in performance when faults are injected. It should be noted that in the results below the actuator load is a moving mass, M, (see fig 7). Application of other loads, such as applied external forces, was found to have very little effect on the overall results.

\subsection{Step response}

A $0.06 \mathrm{~m}$ step signal will be used to test the step response of the SP configuration under both control structures. Fig. 13 shows the performance of the HRA using classical voltage-driven controller under healthy and locked up situations. In the figure, the key parameter is the actuator position (third from top) which responds to a step in set-point from $0 \mathrm{~m}$ to $0.06 \mathrm{~m}$ at a time of 0seconds. It can be seen that the performance is slightly degraded (in terms of rise and settling times), but that the set-point is achieved even in the case of the locked fault. Other performances in both time and frequency domain (for this fault case and others) are summarised in Table 2. 

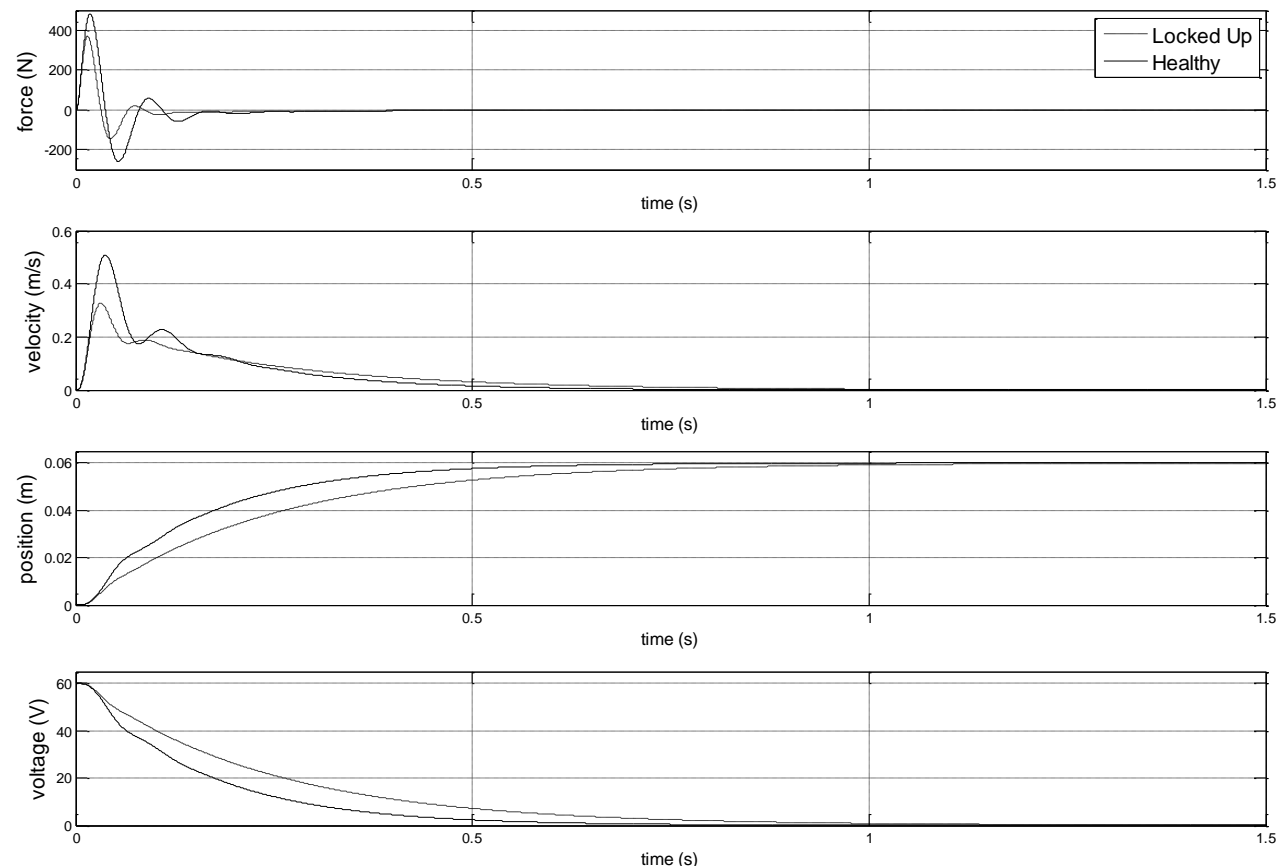

Fig. 13 Performance under healthy and locked up situation using voltage-driven controller

TABLE 2

Performance of SP configuration with voltage-driven (up) and current-driven (down) control structure

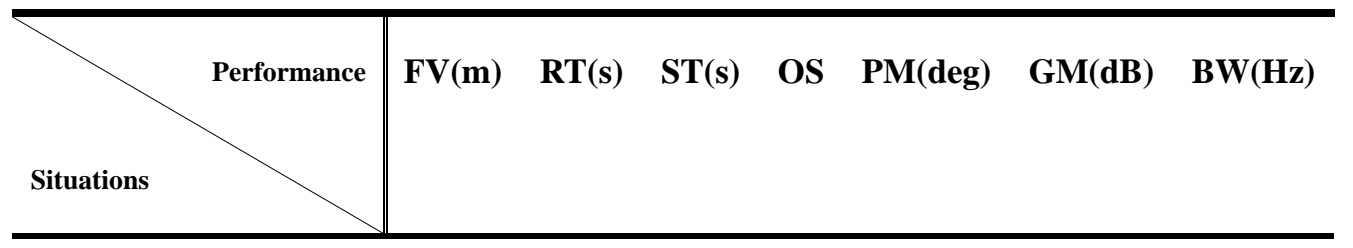

Voltage-driven

\begin{tabular}{l||cccccccc}
\hline Healthy & 0.06 & 0.35 & 0.60 & $0 \%$ & 83.7 & 11.4 & 1 \\
\multirow{2}{*}{$\begin{array}{c}\text { Overheating } \\
\text { Open circuit }\end{array}$} & 0.06 & 0.30 & 0.58 & $0 \%$ & 85.1 & 18 & 1.33 \\
Backlash & 0.06 & 0.36 & 0.61 & $0 \%$ & 86.7 & 12.1 & 1 \\
& 0.06 & 0.35 & 0.60 & $0 \%$ & 86.9 & 17.5 & 1 \\
Lockup & 0.06 & 0.54 & 0.91 & $0 \%$ & 88 & 24.1 & 0.69 \\
\hline
\end{tabular}




\begin{tabular}{c||cccccccc}
\hline Healthy & 0.06 & 0.39 & 0.62 & $0 \%$ & 80.8 & 23.5 & 1 \\
Overheating & 0.06 & 0.44 & 0.74 & $0 \%$ & 82.1 & 24.4 & 0.88 \\
Open circuit & 0.06 & 0.40 & 0.62 & $0 \%$ & 80.6 & 17.9 & 0.96 \\
Backlash & 0.06 & 0.40 & 0.62 & $0 \%$ & 80.8 & 23.5 & 1 \\
Lockup & 0.06 & 0.60 & 0.99 & $0 \%$ & 83.8 & 30.0 & 0.64 \\
\hline
\end{tabular}

KEY: FV is Final value of position, RT is Rise time, ST is Settling time, and OT is overshoot, GM is Gain margin, PM is Phase margin, BW is Band width.

From the table listed above, firstly, the HRA can reach the reference position under all fault situationsand the degradation when faults are injected is not judged to be significant. For both controllers, the worst case is the lockup fault. With the level of redundancy being considered here, this kind of fault is more serious than the others because it can not be solved on a signal level. When this happens to an individual actuator, it only can be accommodated through redundancy rather than through a robust controller; hence degradation can be seen and expected.

For these two control structures, although performance degradation can be seen when faults are injected, it is hard to say which one is more sensitive to faults. It is possible to observe that there are bigger changes in bandwidth when faults occur in the voltage control case. However it's settling time is affected less than that of the current control. The changes in rise time are of a similar magnitude in both cases. 


\subsection{Ramp response}
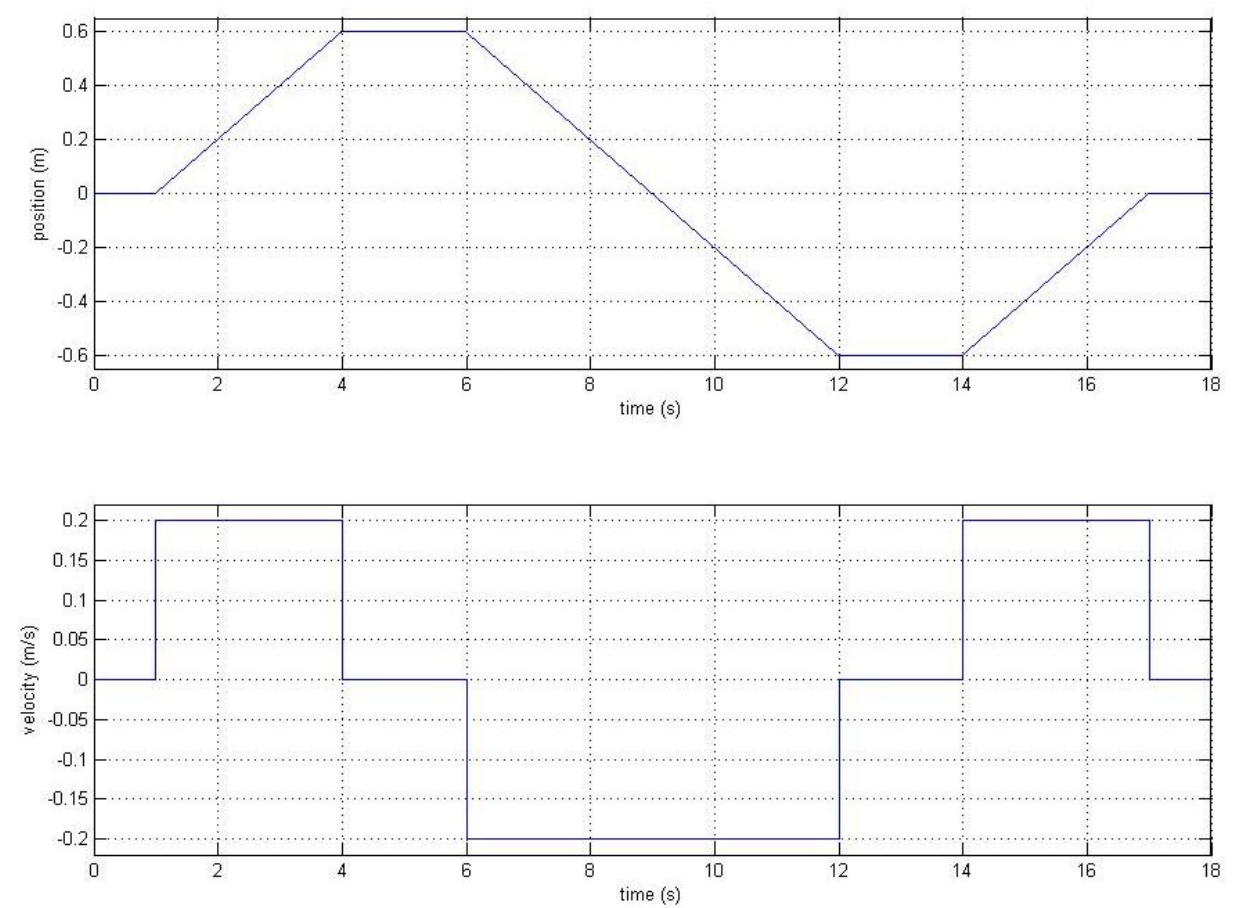

Fig. 14 Position and velocity input

A ramp position input is created between $-0.6 \mathrm{~m}$ and $0.6 \mathrm{~m}$ with a slope of $0.2 \mathrm{~m} / \mathrm{s}$. The velocity input is a step signal, while the position input is a ramp signal. The HRA uses the same controllers designed for the fault-free case via position feedback information. The command signal is shown in Fig. 14.

The coefficient of determination for both position and velocity output is calculated in each case. This is a statistical metric that measures how well the output tracks the input. It is given by the following equation:

$r t^{2}=1-\left(\frac{e}{y_{d}-\operatorname{mean}\left(y_{d}\right)}\right)^{2}$,

where $e$ is the error between the input and output, $y_{d}$ is the input value. 
TABLE 3

Determination value for position and velocity of SP configuration with voltage-driven and current-driven control structure
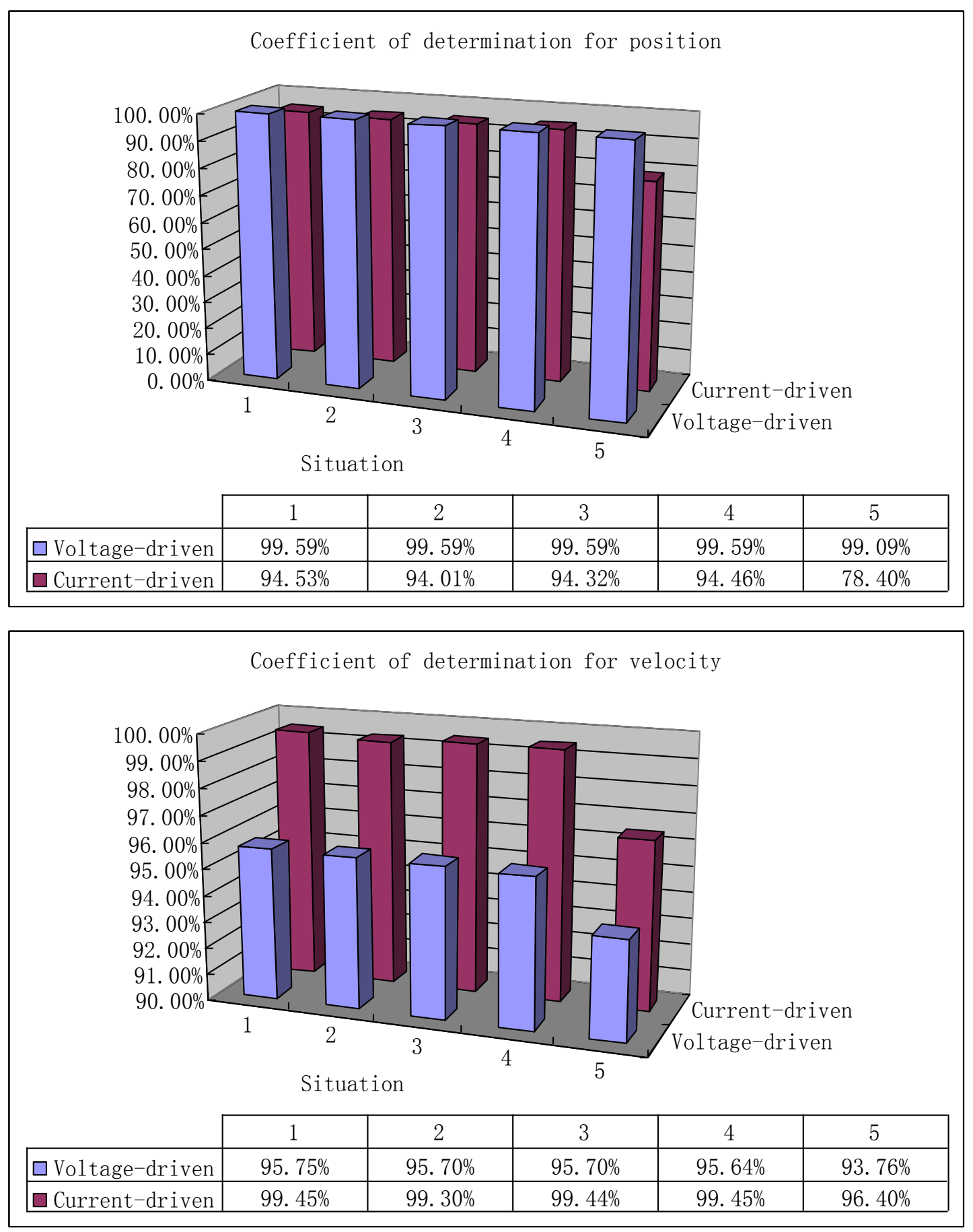

The results of determination values are listed in Table. 3 using two bar diagram to make it more visual. 
Again, the Bottom 1 sub-actuator element is selected as the injected by faults. Five situations are listed.

First is Healthy situation, and the others are Overheating, Open circuit, Backlash, and Lockup situations separately. Inspection of table 3 reveals that performance degradation can be found when faults are injected and the worst case is for the lockup fault, especially using current-driven control structure. The results also suggest that the voltage-driven structure is more suitable for position tracking while the current-driven structure is more suitable for velocity tracking (because a bigger value of determination coefficient for position is obtained in the voltage-driven structure while a bigger value of determination coefficient for velocity is found with the current-driven structure). The reason is due to the fast current inner loop added to the current-driven control structure. Since the current is a higher order state inside the HRA so that it makes the HRA easier to track a velocity or/and force command when current inner loops added. From this point of view, it can be concluded that the current-driven control structure is not as effective for position tracking purpose while the voltage-driven control structure can work on both position and velocity tracking purposes although a better performance can be obtained for a position tracking purpose.

\section{Conclusion}

The HRA is a novel actuator concept which provides the ability of fault tolerance without reconfiguration because it comprises a relatively large number of sub-actuation elements. This paper presented a linear model of a relatively simple two by two configuration of the HRA, as well as two candidate controllers designs based on a classical approach. The model has been set up and tested in MATLAB/Simulink. Four fault scenarios, including electrical and mechanical faults, were modelled to 
test the fault tolerance ability of the HRA. The simulation results show some performance degradation occurs when faults are injected into one of the actuation elements, but the HRA can still complete the whole function without any reconfiguration at either mechanical or signal level. According to all these results, it's straightforward to conclude that the high redundancy actuator is relatively easy to control in both healthy and faulty situations. Both control structures proposed appear to be suitable for the task and there is little to choose between them.

For future work, extensions include testing the actuator with a large range of faults. More importantly the two by two configuration will be extended to four by four (and ultimately to higher dimensions), to ensure that the concept scales up. It should be noted that the models used in this study are linear, whilst actuators such as this do not generally exhibit high degrees of non-linearity, the effects of such issues and measurement noise have not been considered so far. To address this, an experimental HRA assembly based on the two by two SP configuration is currently being built together with the associated control system, which will help to confirm the practical viability of the HRA under both healthy and faulty situations. In the longer term it will also be important to consider possible applications for HRA and to investigate which design methods and actuation technologies are most appropriate for their manufacture.

\section{Acknowledgements}

The authors would like to thank the Engineering and Physical Sciences Research Council (EPSRC) for funding this project under grant reference EP/D078350/1, as well as the successful cooperation with the Systems Engineering Innovation Centre (SEIC) and SMAC Europe ltd. 


\section{References}

[1] R. Patton (1997). Fault Tolerant Control: The 1997 Situation. SAFE-PROCESS'97, IFAC Symposium on fault detection, supervision and safety, Kingston Upon Hull, U.K.

[2] D. Briere, C. Favre, and P. Traverse (1995). A family of Fault Tolerant Systems: Electrical flight controls from Airbus A320/A330/A340 to future military transport aircraft. Microprocessors and Microsystems, Volume 19, Number 2, pp. 75-82

[3] Terry. Ford (1998). Actuation systems development. Aircraft Engineering and Aerospace Technology, Volume 70, Number 4, pp. 265-270

[4] R. Isermann (1984). Process fault detection based on modelling and estimation methods - A survey. Automatica, Volume 20, No. 4, pp. 387-404

[5] R. Isermann, R. Schwarz, and S. Stolzl (2002). Fault-tolerant drive-by-wire systems. IEEE Control

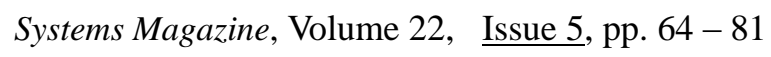

[6] G. Liu, and D. Wang (2004). Active fault tolerant control with actuation reconfiguration. IEEE Transactions on Aerospace and Electronic Systems, Vol. 40, No. 3 pp.1110 - 1117

[7] J. Lunze, and T. Steffen (2006). Control reconfiguration after actuator failures using disturbance decoupling methods. IEEE Transactions on Automatic Control, Vol. 51, No. 10, pp. $1590-1601$

[8] H. Noura, D. Theilliol, and D. Sauter (2000). Actuator fault-tolerant control design: demonstration on a three-tank-system. International Journal of Systems Science, Vol. 31, No. 9, pp. $1143-1155$

[9] G. Tao, S. M. Joshi, and X. Ma (2001). Adaptive state feedback and tracking control of systems with actuator failures. IEEE Transactions on Automatic Control, Vol. 46, No. 1, pp. 78 - 95 
[10] W. Chen and J. Jiang (2005). Fault-tolerant control against stuck actuator faults. IET Control Theory \& Applications, Vol. 152, No. 2, pp $138-146$

[11] Y. Zhang, J. Jiang (2003). Fault tolerant control system design with explicit consideration of performance degradation. IEEE Transactions on Aerospace and Electronic Systems, Vol. 39, No. 3 , pp. $838-848$

[12] Mclennan Servo Supplies Ltd. http://www.mclennan.co.uk/

[13] Ian Pratt (1996). Active suspension applied to railway train, PhD thesis in Loughborough University, Section 1, pp. $59-66$

[14] J. H. Baek, Y. K. Kwak, and S. H. Kim (2003). On the frequency bandwidth change of a servo system with a gear reducer due to backlash and motor input voltage. Archive of Applied Mechanics, Volume 73, pp. 367-376

[15] Ch. M. Hajiyev, and F. Caliskan (2001). Integrated sensor/actuator FDI and reconfigurabl3 control for fault-tolerant flight control system design, The Aeronautical Journal (2001), pp. 525 - 533

[16] Slotine, J.E., Li, W. (1991). Applied nonlinear control. USA, Prentice-Hall

[17] X. Du, R. Dixon, R. M. Goodall, and A. C. Zolotas (2006). Assessment of strategies for control of high redundancy actuators, Proceedings of the ACTUATOR 2006, Bremen, Germany

[18] X. Du, R. Dixon, R. M. Goodall, and A. C. Zolotas (2006). Modelling and control of a highly redundant actuator, International Control Conference 2006, Glasgow, U.K. 


\section{List of Figures}

1. Scheme of fault-tolerant control system with supervision subsystem (Patton, 1997)

2. Several possible configurations of the high redundancy actuator

3. Equivalent model of individual electro-mechanical actuator

4. Free body diagram of individual electro-mechanical actuator

7. Equivalent model of two by two SP configuration

\section{List of Tables}

2. Performance of SP configuration with voltage-driven and current-driven control structure

3. Determination value for position and velocity of SP configuration with voltage-driven and current-driven control structure 